\title{
A Computational Model of the Cerebellum to Simulate Cortical Degeneration During a Pavlovian Associative Paradigm
}

\author{
A. Geminiani ${ }^{1}$, A. Antonietti ${ }^{1}$, C. Casellato ${ }^{1}$, E. D’Angelo ${ }^{2,3}$ and A. Pedrocchi ${ }^{1}$ \\ ${ }^{1}$ Department of Electronics, Information and Bioengineering, Politecnico di Milano, \\ Neuroengineering and Medical Robotics Laboratory, Milano, Italy \\ ${ }^{2}$ Brain Connectivity Center, IRCCS Istituto Neurologico Nazionale C. Mondino, Pavia, Italy \\ ${ }^{3}$ Department of Brain and Behavioral Sciences, University of Pavia, Pavia, Italy
}

\begin{abstract}
Thanks to the development of realistic neural models, computational neuroscience is becoming fundamental to understand the complex functioning of the human brain. Several studies have demonstrated the role of computational models in revealing the links between the low-level activity at multiple neural scales and human-like behaviors, under physiological conditions. However, limited resources have been employed in applying computational neuroscience to the understanding of brain diseases. In this work, we focused on the cerebellum that has a crucial role in driving learning and timing of motor commands during associative tasks. We provided a preliminary analysis on the use of a computational model to investigate a type of cerebellar ataxia that involves cortical degeneration. Starting from an optimized realistic Spiking Neural Network representing the cerebellar microcircuit, we applied specific local modifications to mimic the same damage as in patients. Then, we analyzed the behavior generated by the low-level neural activity of the network during a Pavlovian associative task. We were able to reproduce the same impaired behavior shown in human tests, characterized by a decreased capability to associate the provided stimuli. Moreover, the changes in the simulated neural activity agreed with the findings from neural recordings in animals affected by the same pathological condition. Therefore, we demonstrated that our model could be used also to represent cerebellar pathologies, with the possibility to link damages at neural level to compromised behaviors, which is one of the main actual challenges in neuroscience.
\end{abstract}

Keywords- Cerebellum, Computational Neuroscience, Genetic Algorithm, Pathology, Spiking Neural Network.

\section{INTRODUCTION}

In these years, much effort in the neuroscience community is being put on the development of new tools, coming from the computer science and the engineering world, to explore and understand the complex mechanisms of the brain functioning [1, 2]. The two brain initiatives, both in Europe [3] and in US [4], are aiming to generate physical and conceptual tools to determine how human brain functions at different scales: from molecules to behaviors and thoughts, linking what is known about single neurons to the high-level brain function. These new tools aimed at establishing a holistic understanding of how the dynamic activities in the brain generate actions, consciousness and cognition.

Computational models are considered exciting opportunities to make predictions, starting from the neurophysiological evidences, on the macroscopic and microscopic behavior of the brain [5]. This work circumscribes the application of computational models to the cerebellum only. Both animal and human studies provided evidence that the cerebellum plays an important role in a temporal associative paradigm, the Eye Blink Conditioning (EBC) [6]. Extensive research has been performed to elucidate the various contributions of the different layers of the cerebellum (e.g. the cerebellar cortex, the granular layer and the deep nuclear layer), in the acquisition, extinction and retention of conditioned eye blink responses [7].

The Pavlovian Eye Blink association is learned along with repeated paired presentation of stimuli, a Conditioned Stimulus (CS, a tone) followed by an Unconditioned Stimulus (US, an air-puff, eliciting the eye blink reflex). The cerebellum learns to produce a Conditioned Response (CR, like an eye blink) anticipating the US onset [8].

The cerebellum employs long-term synaptic modifications to learn and store information: these mechanisms are Long-Term Depression (LTD) and LongTerm Potentiation (LTP). The plasticity at the Parallel Fibers-Purkinje Cells (PFs-PCs) synapses has classically been assumed to learn the temporal association during the EBC, but it cannot account for the multiple time-scales of cerebellar learning. Plastic mechanisms involving other synapses were hypothesized $[9,10]$ and a cerebellum-inspired model was developed [11], consisting of a realistic Spiking Neural Network (SNN). In previous works [12-16], the cerebellar model was tested in EBC simulations and it was able to reproduce realistic behaviors, similar to the human ones.

In this study, we aimed at exploiting the detailed computational model to mimic a pathology of the cerebellum, thus providing a proof of concept of the 
application of computational neuroscience in medicine to understand brain diseases [17]. In fact, due to the high complexity of neural circuits, it is becoming necessary to develop new techniques that could be used together with traditional methods to reveal the mechanisms causing pathologies, so as to overcome the limits of the current investigation techniques.

We focused on the cerebellar cortex, since a damage to this area of the cerebellum has been associated with specific pathologies $[18,19]$. In this case, the EBC paradigm is a common tool to investigate the impairment. In fact, cortical neurons have an inhibitory action on the cerebellar output, which can be modulated, thanks to synaptic plasticity with a precise timing, to generate the CR [8]. Therefore, a cortical damage results in a compromised conditioning during associative tasks, as demonstrated also by Dimitrova and colleagues in [20], here considered as the reference experimental case.

After an automated tuning of the cerebellar model's parameters to replicate the healthy behavior, we "impaired" the cerebellar model, reducing the number of PCs. The modified network generated a compromised behavior, similar to the cerebellar patients' one. With this computational approach, we were able to analyze the underlying mechanisms of observed behaviors, both in healthy and in altered conditions.

\section{MAterials AND METHODS}

\section{A. Cerebellar Model}

In order to reproduce physiological and pathological conditions during EBC, we used an open source platform modeling the cerebellum as a realistic SNN [21, 22]. Specifically, the simulator was based on the event-driven simulation method and it used pre-compiled Look-Up Tables to speed up the computation of neural states [23]. We performed all the tests on a desktop PC (Intel ${ }^{\circledR}$ Xeon ${ }^{\circledR}$ CPU E5-1620 v2 @3.70 GHz with 32 GB of RAM and Windows 7 as Operating System). We customized the network in order to have population size and connection ratios inspired to the physiological cerebellar features. We then provided the SNN with specific input signals to simulate the EBC paradigm [15]. Therefore, the resulting model included 6480 total neurons (Figure 1): 300 Mossy Fibers (MFs) conveyed the CS to 6000 Granular Cells, giving a sparse encoding of the input and then contributing to the passage-of-time representation, which is fundamental in EBC [24]; the Granular Cells signal converged to 72 PCs through the Parallel Fibers, while the PCs also received the US teaching signal from 72 Inferior Olive (IOs) neurons.
The sole output of the cerebellar network was provided by the 36 DCNs cells, receiving an excitatory signal from MFs and an inhibitory signal from PCs.

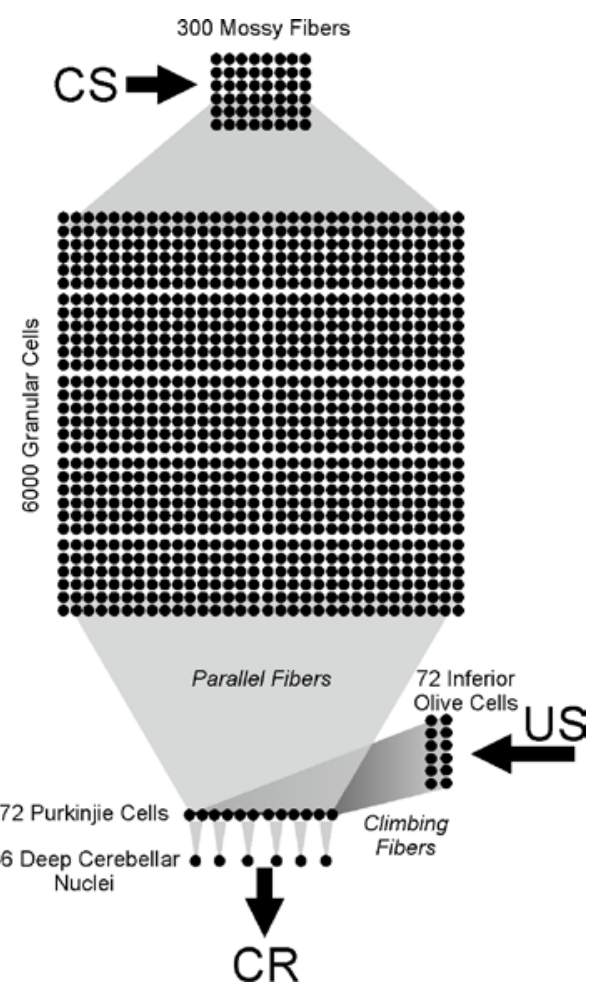

Fig. 1 SNN architecture, with different groups of cells and relative input (i.e. CS and US) and output (i.e. CR) signals.

During acquisition trials, both CS and US were provided to the model, as random spike patterns with physiological firing rates to the MFs and the IOs, respectively. On the other hand, in the extinction trials the CS was the only input to the network. The CR was detected based on the analog signal obtained after decoding the DCNs spiking activity. In particular, a CR was generated at time $t_{C R}$ when the DCNoutput signal overcame the threshold baseline+offset, being baseline the mean value of the signal in the first time window of each trial and offset a constant value. In addition, two further constraints were verified: the CR occurred in the $200 \mathrm{~ms}$ interval before US onset and the DCNoutput signal reached the threshold after a rapid increase of its value [25]; to fulfill the second condition, we imposed:

$$
\frac{\text { DCNoutput }\left(\mathrm{t}_{\mathrm{CR}}\right)}{\text { mean }\left(\text { DCNoutput }\left(\mathrm{t} \leq \mathrm{t}_{\mathrm{CR}}\right)\right)} \geq 3
$$

Synaptic plasticity contributed to modify the activity of specific neural populations and then to modulate the output signal in order to achieve conditioning. For this purpose, the 
network was endowed with 3 plasticity sites at both cortical and nuclear level [14, 26]. Plasticity at PFs-PCs synapses was responsible for fast learning and it was modeled as LTP and LTD modulated by the IOs teaching signal. On the other hand, nuclear plasticity at MFs-DCNs connections was modeled as LTP and LTD triggered by PCs, while Spike Time-Dependent Plasticity was used for PCs-DCNs synapses. Specific learning rules were used for the three plasticity sites [15].

\section{B. Optimization algorithm}

As the overall system was quite complex and included several parameters, a Genetic Algorithm (GA) was selected to tune the model, due to the demonstrated success of this technique in optimizing the weights and the parameters of neural networks [27]. The algorithm was developed in MATLAB environment, using the Parallel Computing Toolbox to speed up the whole process. Each generation included a population of 12 individuals, whose genes were the LTP and LTD rates for the learning rules and the initial weights at each plasticity site: $\mathrm{LTP}_{1}$ and $\mathrm{LTD}_{1}$ for the PFsPCs connections, $\mathrm{LTP}_{2}$ and $\mathrm{LTD}_{2}$ for the MFs-DCNs synapses, $\mathrm{LTP}_{3}$ and $\mathrm{LTD}_{3}$ for the PCs-DCNs connections, and weight $0_{P F P C}$, weight $0_{M F D C N}$, weights $0_{P C D C N}$.

The performance of each individual was evaluated based on the outcome of EBC simulations. The protocol for the associative task included 2 consecutive sessions of 100 acquisition trials and 30 extinction trials, with an Inter Stimulus Interval (ISI) $=440 \mathrm{~ms}, \mathrm{CS}=540 \mathrm{~ms}$, US $=100$ ms co-terminating with CS (“delay EBC”). Based on human neurophysiological data on EBC [28], the maximum fitness value (i.e. 1) was assigned to the individuals reaching $70 \%$ of CRs during acquisition and $30 \%$ of CRs during extinction of both sessions. After computing the fitness for each individual, selection, crossover and mutation were performed. The genetic operators were chosen so as to achieve elitism, exploration and exploitation of the solution space, which are fundamental principles in the GA theory.

When one of the stop criteria (i.e. reaching a maximum number of generations or having no improvements of the maximum fitness for a fixed number of generations) was met, the optimal parameters were computed as the mean value of the genes of all the 1-fitness individuals throughout the evolution process.

Then, in order to verify the proper physiological functioning of plasticity mechanisms, we performed simulations of the same protocol also with the 1-plasticity version of the network, where only PFs-PCs synapses underwent LTP and LTD, using the same optimal parameters [16].
We evaluated the outcome of the model, i.e. the \%CR in blocks of 10 trials, and comparing the results in the 2 sessions of the 1-plasticity and 3-plasticity simulations. In particular, we were interested in deepening the differences of the two models in long term, i.e. during the second session after the long acquisition and the extinction phases of session 1 .

\section{Simulation of cortical degeneration}

Starting from the optimized and validated physiological model, we modified specific low-level properties in order to reproduce pathological conditions.

We referred to an experimental study on cerebellar patients suffering from some types of Spinocerebellar Ataxia [20], characterized by a lesion to the cerebellar cortex. To reproduce the same damage in our model, we removed 15 PCs from the network, corresponding to $20 \%$ of the total PCs. We then performed EBC simulations with 36 different random templates of PCs removal so as to obtain stable results. We used the same protocol as in the reference study: 10 blocks of 10 trials including 9 CS-US trials and one CS-alone trial, with ISI $=440 \mathrm{~ms}$, CS lasting $540 \mathrm{~ms}$, US lasting $100 \mathrm{~ms}$ and co-terminating with CS.

The outcomes of the optimal physiological and pathological models were compared through the representation of the total percentage of CRs during the simulations and the \%CR in each 10-trial block. We used the mean and Standard Error (SE) among the 36 pathological simulations, as performance parameters, like in the reference study. Then, the results were compared to the outcome of experiments on patients, in order to prove the capability of the model to reproduce the impaired behavior that typifies PCs lesion.

We also analyzed the low-level activity of the network, to achieve a deeper insight into the altered mechanisms involved in the pathological behavior. We represented the DCNoutput signal in a late acquisition trial for both physiological and pathological simulations; so we could make the comparison with the results of animal recordings that investigated the role of the cerebellar cortex during EBC [8]. Moreover, we evaluated the spiking activity of PCs and DCNs, which are the neural populations influenced by synaptic plasticity. For the healthy case and the pathological example, we computed the number of spikes in time-bins of $10 \mathrm{~ms}$ during each trial, for the whole simulation protocol. 


\section{REsUlts}

\section{A. Optimized physiological model}

The starting point of our analysis was the optimized realistic model in physiological conditions. After 100 generations without any improvement of the maximum fitness, the GA stopped and we obtained the final parameters as the mean values of the 1-fitness individuals' genes. This way, we selected the solution in an intermediate optimal region of the search space. The optimization strategy also allowed to automate the tuning procedure, sparing time and resources.

Thanks to the further check with the 1-plasticity version of the model, we could confirm the validity of the obtained parameters. In fact, the trend of \%CR agreed with the results obtained on the same protocol with a similar model [15]. As shown in Figure 2, the consolidation role of nuclear plasticity appeared in session 2, where the 3-plasticity network achieved a faster acquisition and a higher \%CR [28].

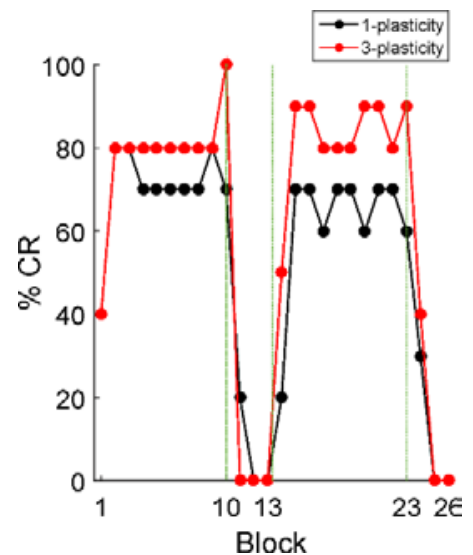

Fig. 2 \%CR in blocks of 10 trials during protocol after optimization, with the 1-plasticity (black line) and the 3-plasticity (red line) networks.

\section{B. Pathological model in case of cortical degeneration}

The results of simulations with 15 removed PCs demonstrated the high potential of computational models in reproducing and understanding pathological conditions.

We showed that in case of a cortical lesion, the mean total percentage of CRs was significantly lower than in the healthy case and conditioning did not occur neither during late acquisition, with a maximum $10 \%$ of average \%CR (Figure 3A). This impaired behavior perfectly agreed with the outcome of tests in cerebellar patients [20]. Indeed, they obtained the same mean total percentage of CRs and a similar non-increasing trend of \%CR, characterized by a mean value of about $10 \%$ throughout the whole protocol (Figure 3B).

In healthy conditions, they reported an increase of \%CR throughout the protocol, reaching a lower total \%CR than in our simulations. This discrepancy depended on the fact that our simulated healthy model had to reach the $70 \%$ of CRs during the acquisition phase.
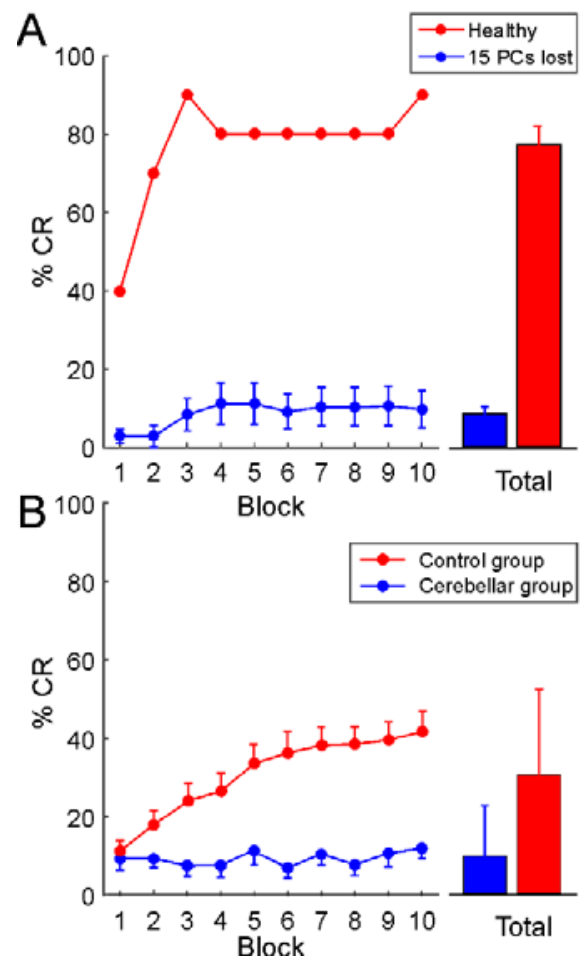

Fig. 3 Comparison between the outcome of simulations (Panel A) and the results in the reference study (Panel B - adapted from [20]). The mean and SE of \%CR in blocks of 10 trials and over the total blocks are reported for the healthy (in red) and pathological (in blue) cases.

In the analysis of the neural changes leading to the behavioral modifications, we were able to verify the same low-level outcome as in animal experiments that investigated PCs loss. The physiological DCNoutput during EBC was characterized by a peak corresponding to the CR and a null value elsewhere. When removing some PCs, the shape of the DCNoutput was modified: the signal had a higher value during the whole trial and it was not possible to identify a significant sharp peak generating the CR (Figure 4). A similar change in the cerebellar output was measured in rabbits after a lesion to the cortex [8].

Going further into this analysis, we found the basic causes for the altered output of the circuit and the compromised behavior, in the variation of PCs and DCNs spiking activity. As shown in Figure 5A, the removal of 
some PCs caused a decrease of their activity along all the trial duration; nevertheless, synaptic plasticity at the functioning PFs-PCs synapses completely inhibited PCs in the time window for CRs, after some acquisition trials.

However, this was not sufficient to generate CRs, because the lack of an efficient time-locked inhibition on the DCNs by the PCs caused the nuclear neurons to increase their baseline activity (Figure 5B). Therefore, the resulting output signal did not fulfill all the requirements to produce a $\mathrm{CR}$, as previously explained.
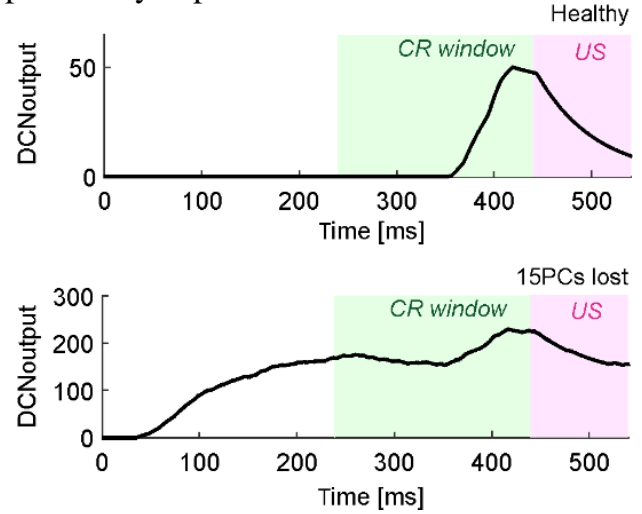

Fig. 4 Representation of the DCNoutput signal during a late acquisition trial, for simulations of the healthy (top) and pathological case (bottom).

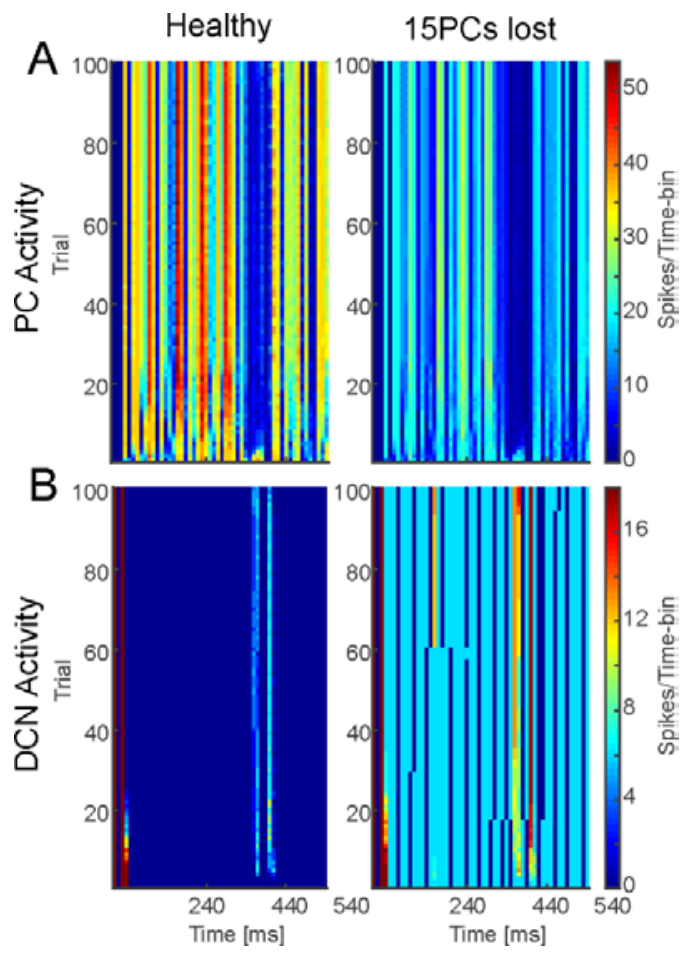

Fig. 5 Spiking activity of PCs (Panel A) and DCNs (Panel B) as the number of spikes in time-bins of $10 \mathrm{~ms}$ for the 100 trials of the protocol, during healthy (on the left) and pathological (on the right) simulations.

\section{Discussion}

Our results demonstrated the potential role of computational neuroscience in investigating brain diseases.

Thanks to the optimization and validation of our realistic cerebellar network through the comparison with previous results, we started from a solid basis to develop a proof of concept for a new tool in medicine. We were able to apply a localized lesion to the cerebellar circuit and then study the effects on associative learning and underlying neural activity. In fact, the analysis of the low-level behavior of the SNN (e.g. the shape of the DCNoutput or the changes in the firing rates patterns of PCs and DCNs) in the two conditions, explained the causes of the high-level misbehavior. Then, we could generalize our results to other cerebellar pathologies, involving the PCs damage reproduced in our network. For example, a decrease in the PCs number has been associated with fetal alcohol exposure [19] or with the age-related neural loss which is typical of Alzheimer's Disease patients [18].

Further analysis should be performed to predict the role of neural plasticity in this pathological condition. In fact, synaptic modifications have been proved crucial for cerebellum-driven physiological motor learning [9], and they could have a key role in developing compensatory mechanisms after a damage. Moreover, other cerebellar diseases could be considered in order to demonstrate the robustness of the current approach as an instrument to reproduce the effect of different lesions in the cerebellar circuit. Finally, an update of the network (e.g. increase the number of neurons and including plasticity at further sites) will become necessary to improve the realism of the model and use it as a diagnostic tool.

In summary, promising applications of our method include the use in neuroscience to predict the behavior under different conditions and to deeply understand brain diseases, by making and verifying hypotheses on pathological mechanisms. Finally, realistic computational models could be used to simulate the effect of drugs and suggest possible treatments for diseases.

\section{v. Conclusions}

In this work, we developed a realistic pathological model of the cerebellum with a decreased number of PCs that properly reproduced the altered behavior observed in ataxic patients. Moreover, also the modified underlying mechanisms agreed with the results from neural recordings in animals characterized by the same cortical lesion. Then, we could shed light on the changed neural activity leading 
to the high-level misbehaviors, which is not possible during in vivo experiments.

Therefore, given the promising results, the current approach paves the way to the development of new branches of computational neuroscience investigating brain diseases.

\section{ACKNOWLEDGMENT}

This work was supported by grants of European Union: REALNET (FP7-270434) and Human Brain Project (HBP 604102) and HBP-Regione Lombardia.

\section{CONFLICT OF INTEREST}

The authors declare that they have no conflict of interest.

\section{REFERENCES}

1. Redolfi A, Bosco P, Manset D, Frisoni GB (2013) Brain investigation and brain conceptualization. Funct Neurol 28:175. doi: 10.11138/FNeur/2013.28.3.175

2. Markram H (2006) The blue brain project. Nat Rev Neurosci 7:15360. doi: $10.1038 / \mathrm{nrn} 1848$

3. Markram H (2012) The Human Brain Project. Sci Am 306:50-55. doi: 10.1038/scientificamerican0612-50

4. Insel TR, Landis SC, Collins FS (2013) The NIH BRAIN Initiative. Science (80- ) 340:687-688. doi: 10.1126/science.1239276

5. D’Angelo E, Solinas S, Garrido JA, et al. (2013) Realistic modeling of neurons and networks: towards brain simulation. Funct Neurol 28:153-166. doi: 10.11138/FNeur/2013.28.3.153

6. Bracha V, Zhao L, Irwin KB, Bloedel JR (2000) The human cerebellum and associative learning: dissociation between the acquisition, retention and extinction of conditioned eyeblinks. Brain Res 860:87-94. doi: 10.1016/S0006-8993(00)01995-8

7. Hansel C, Linden DJ, D’Angelo E (2001) Beyond parallel fiber LTD: the diversity of synaptic and non-synaptic plasticity in the cerebellum. Nat Neurosci 4:467-75. doi: 10.1038/87419

8. Medina JF, Garcia KS, Nores WL, et al. (2000) Timing mechanisms in the cerebellum: testing predictions of a large-scale computer simulation. J Neurosci 20:5516-25.

9. D’Angelo E, Mapelli L, Casellato C, et al. (2015) Distributed Circuit Plasticity: New Clues for the Cerebellar Mechanisms of Learning. The Cerebellum. doi: 10.1007/s12311-015-0711-7

10. Medina JF, Mauk MD (1999) Simulations of Cerebellar Motor Learning: Computational Analysis of Plasticity at the Mossy Fiber to Deep Nucleus Synapse. J Neurosci 19:7140-7151.

11. Garrido JA, Ros E, D’Angelo E (2013) Distributed synaptic plasticity controls spike-timing: predictions from a cerebellar computational model. BMC Neurosci 14:P81. doi: 10.1186/1471-2202-14-S1-P81

12. Casellato C, Antonietti A, Garrido JA, et al. (2015) Distributed cerebellar plasticity implements generalized multiple - scale memory components in real - robot sensorimotor tasks. Front Comput Neurosci. doi: 10.3389/fncom.2015.00024

13. Casellato C, Antonietti A, Garrido JA, et al. (2014) Distributed cerebellar plasticity implements multiple-scale memory components of Vestibulo-Ocular Reflex in real-robots. In: 2014 5th IEEE RAS/EMBS Int. Conf. Biomed. Robot. Biomech., pp
813-818. doi. 10.1109/BIOROB.2014.6913879

14. Antonietti A, Casellato C, Garrido JA, et al. (2015) Spiking cerebellar model with multiple plasticity sites reproduces eye blinking classical conditioning. In: 2015 7th Int. IEEE/EMBS Conf. Neural Eng. pp 296-299. doi: 10.1109/NER.2015.7146618

15. Antonietti A, Casellato C, Garrido JA, et al. (2016) Spiking Neural Network With Distributed Plasticity Reproduces Cerebellar Learning in Eye Blink Conditioning Paradigms. IEEE Trans Biomed Eng 63:210-9. doi: 10.1109/TBME.2015.2485301

16. Antonietti A, Casellato C, Geminiani A, et al. (2015) Healthy and pathological cerebellar Spiking Neural Networks in VestibuloOcular Reflex. In: 2015 37th Annu. Int. Conf. IEEE Eng. Med. Biol. Soc. pp 2514-2517. doi: 10.1109/EMBC.2015.7318903

17. Markram H (2013) Seven challenges for neuroscience. Funct Neurol 28:145-151. doi: 10.11138/FNeur/2013.28.3.145

18. Woodruff-Pak DS, Papka M, Romano S, Li YT (1996) Eyeblink classical conditioning in Alzheimer's disease and cerebrovascular dementia. Neurobiol Aging 17:505-512. doi: 10.1016/S0197-4580(96)00070-X

19. Jacobson SW, Stanton ME, Dodge NC, et al. (2011) Impaired Delay and Trace Eyeblink Conditioning in School-Age Children With Fetal Alcohol Syndrome. Alcohol Clin Exp Res 35:250-264. doi: 10.1111/j.1530-0277.2010.01341.x

20. Dimitrova A, Gerwig M, Brol B, et al. (2008) Correlation of cerebellar volume with eyeblink conditioning in healthy subjects and in patients with cerebellar cortical degeneration. Brain Res 1198:73-84. doi: 10.1016/j.brainres.2008.01.034

21. Carrillo RR, Ros E, Boucheny C, Coenen OJMD (2008) A real-time spiking cerebellum model for learning robot control. Biosystems 94:18-27. doi: 10.1016/j.biosystems.2008.05.008

22. Casellato C, Antonietti A, Garrido JA, et al. (2014) Adaptive Robotic Control Driven by a Versatile Spiking Cerebellar Network. PLoS One 9:e112265. doi: 10.1371/journal.pone.0112265

23. Ros E, Carrillo RR, Ortigosa EM, et al. (2006) Event-driven simulation scheme for spiking neural networks using lookup tables to characterize neuronal dynamics. Neural Comput 18:2959-93. doi: 10.1162/neco.2006.18.12.2959

24. Yamazaki T, Tanaka S (2007) The cerebellum as a liquid state machine. Neural Networks 20:290-297. doi: 10.1016/j.neunet.2007.04.004

25. Gerwig M, Hajjar K, Dimitrova A, et al. (2005) Timing of conditioned eyeblink responses is impaired in cerebellar patients. J Neurosci 25:3919-31. doi: 10.1523/JNEUROSCI.026605.2005

26. D'Angelo E (2014) The organization of plasticity in the cerebellar cortex: from synapses to control. Prog Brain Res 210:31-58. doi: 10.1016/B978-0-444-63356-9.00002-9

27. Carlson KD, Nageswaran JM, Dutt N, Krichmar JL (2014) An efficient automated parameter tuning framework for spiking neural networks. Front Neurosci 8:10. doi: 10.3389/fnins.2014.00010

28. Monaco J, Casellato C, Koch G, D’Angelo E (2014) Cerebellar theta burst stimulation dissociates memory components in eyeblink classical conditioning. Eur J Neurosci 40:1-8. doi: 10.1111/ejn.12700

Author: Alice Geminiani

Institute: Politecnico di Milano, Neuroengineering and Medical Robotics Laboratory, Department of Electronics, Information and Bioengineering

Street: $\quad$ Piazza Leonardo da Vinci, 32

City: Milano

Country: Italy

Email:_alice.geminiani@polimi.it 